\title{
Enhanced recovery after spine surgery: a systematic review
}

\author{
Mazin Elsarrag, MS, Sauson Soldozy, BA, Parantap Patel, BS, Pedro Norat, MD, \\ Jennifer D. Sokolowski, MD, PhD, Min S. Park, MD, Petr Trrdik, PhD, and \\ M. Yashar S. Kalani, MD, PhD \\ Department of Neurological Surgery, University of Virginia Health System, Charlottesville, Virginia
}

\begin{abstract}
OBJECTIVE Enhanced recovery after surgery (ERAS) is a multidimensional approach to improving the care of surgical patients using subspecialty- and procedure-specific evidence-based protocols. The literature provides evidence of the benefits of ERAS implementation, which include expedited functional recovery, decreased postoperative morbidity, reduced costs, and improved subjective patient experience. Although extensively examined in other surgical areas, ERAS principles have been applied to spine surgery only in recent years. The authors examine studies investigating the application of ERAS programs to patients undergoing spine surgery.
\end{abstract}

METHODS The authors conducted a systematic review of the PubMed and MEDLINE databases up to November 20, 2018.

RESULTS Twenty full-text articles were included in the qualitative analysis. The majority of studies were retrospective reviews of nonrandomized data sets or qualitative investigations lacking formal control groups; there was 1 protocol for a future randomized controlled trial. Most studies demonstrated reduced lengths of stay and no increase in rates of readmissions or complications after introduction of an ERAS pathway.

CONCLUSIONS These introductory studies demonstrate the potential of ERAS protocols, when applied to spine procedures, to reduce lengths of stay, accelerate return of function, minimize postoperative pain, and save costs.

https://thejns.org/doi/abs/10.3171/2019.1.FOCUS18700

KEYWORDS enhanced recovery after surgery; fast-track recovery; spine surgery; scoliosis; minimally invasive surgery; degenerative spine disease

$\mathrm{E}$ NHANCED recovery after surgery (ERAS) is a multidisciplinary, multimodal approach to improving surgical outcomes by using subspecialty- and procedure-specific evidence-based protocols in the care of surgical patients. ${ }^{33}$ Peer-reviewed ERAS protocols are available for various surgical disciplines and procedures. Despite technical differences in these protocols, a common motif is present: minimization and improvement of the stress response. The proposed rationale suggests that by maintaining homeostasis, untoward effects such as postoperative catabolism, pain, and immune dysfunction can be attenuated. ${ }^{25,32,47}$ Components and workflow of a typical ERAS pathway are demonstrated in Fig. 1.

Although Fearon et al. ${ }^{13}$ became the first to formalize such protocols, similar systematic approaches to perioperative care were espoused as early as the 1990s. Initially described as "fast-track surgery" designed to expedite recovery and decrease length of stay (LOS), ERAS has since evolved in both language and approach to focus on optimizing the perioperative experience of surgical patients..$^{25}$ To date, ERAS has been implemented in various surgical specialties. ${ }^{4,5,7,22,30,38,41,45,49,57}$ As the earliest discipline to implement ERAS, colorectal surgery offers a substantial body of literature supporting its benefits. ${ }^{10}$ For example, a meta-analysis of 16 randomized controlled trials (RCTs) of patients undergoing colorectal surgery found a significant reduction of overall morbidity and LOS with use of an ERAS protocol. ${ }^{21}$

ABBREVIATIONS ACDF = anterior cervical discectomy and fusion; $A D=$ accelerated discharge; AIS = adolescent idiopathic scoliosis; ERAS = enhanced recovery after surgery; ERSS = enhanced recovery after spine surgery; LOS = length of stay; PCA = patient-controlled analgesia; PSF = posterior spinal fusion; RCT = randomized controlled trial; TD = traditional discharge; TLIF = transforaminal lumbar interbody fusion

SUBMITTED December 1, 2018. ACCEPTED January 25, 2019.

INCLUDE WHEN CITING DOI: 10.3171/2019.1.FOCUS18700. 


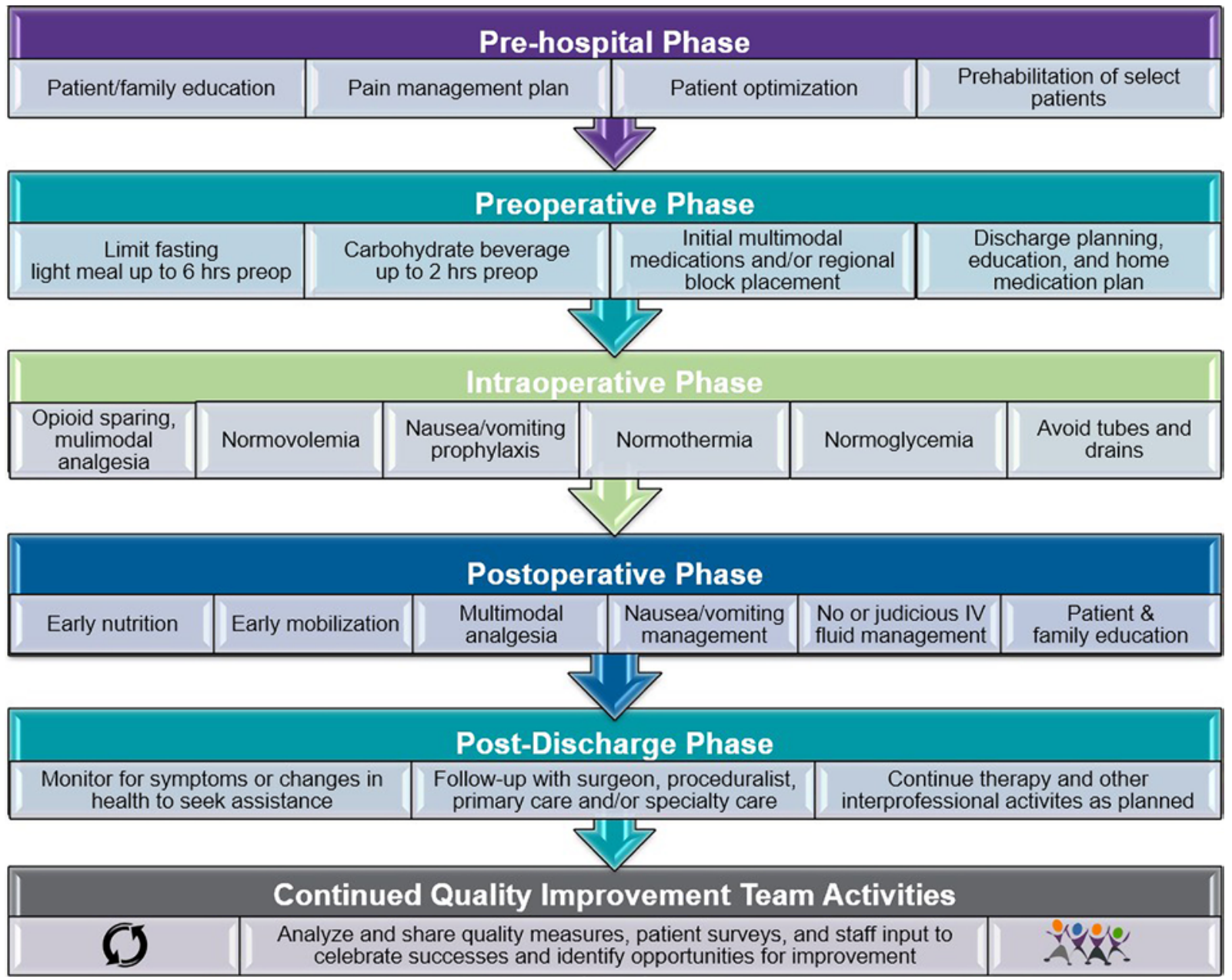

FIG. 1. Components and workflow of a typical ERAS pathway. Copyright American Association of Nurse Anesthetists. Published with permission.

From a healthcare management perspective, ERAS demonstrates financial benefits as well. ${ }^{29}$ A meta-analysis of 10 RCTs consisting of patients undergoing noncolorectal abdominal surgery calculated a mean cost reduction of $\$ 5109.10$ ( $\mathrm{p}<0.001$ ) in the ERAS versus control groups, which was attributed to the combined effect of decreased LOS and no increase in postoperative readmission in ERAS groups. ${ }^{51}$ Furthermore, cost savings often surpass the initial ERAS implementation costs. ${ }^{28,29}$ Patients' positive experiences following an ERAS protocol also indicate subjective benefit. ${ }^{31}$ A study of 95 patients undergoing colorectal surgery observed an increased sense of readiness for discharge (41st to 99th percentile), satisfaction with pain control (43rd to 98th percentile), and likelihood of the patient recommending the hospital (32nd to 89th percentile) in the ERAS group compared to the control group. ${ }^{48}$

Given the apparent benefits of ERAS programs in other surgical disciplines, it is not surprising that its implementation in spine surgery is becoming increasingly common. Wainwright et al. ${ }^{52}$ provided an excellent overview for recovery barriers in the postoperative period with regard to spine surgery, citing that spinal procedures are associated with high amounts of pain, slow return of function, and prolonged hospital stays, among other complications. Al- though they performed a wide literature review examining the adoption of ERAS protocols in spine surgery, the majority of studies identified at the time examined only individual components of ERAS pathways rather than comprehensive programs. Nonetheless, the evidence they reviewed indicated that ERAS principles would likely expedite return to function and minimize postoperative morbidity.

The aim of this systematic review was to identify and examine studies investigating the application of formal ERAS programs to patients undergoing spine surgery.

\section{Methods}

A literature search using PubMed and MEDLINE databases on November 20,2018, was performed using the keywords listed below in combination with Boolean "AND" and "OR" phrases. Titles and abstracts were screened for relevance, and articles describing implementation of enhanced-recovery programs in the context of any spine surgery were included. Bibliographies of works included in the final qualitative synthesis were hand searched for additional relevant studies not found in the original search. Case reports, review articles, or other works without origi- 


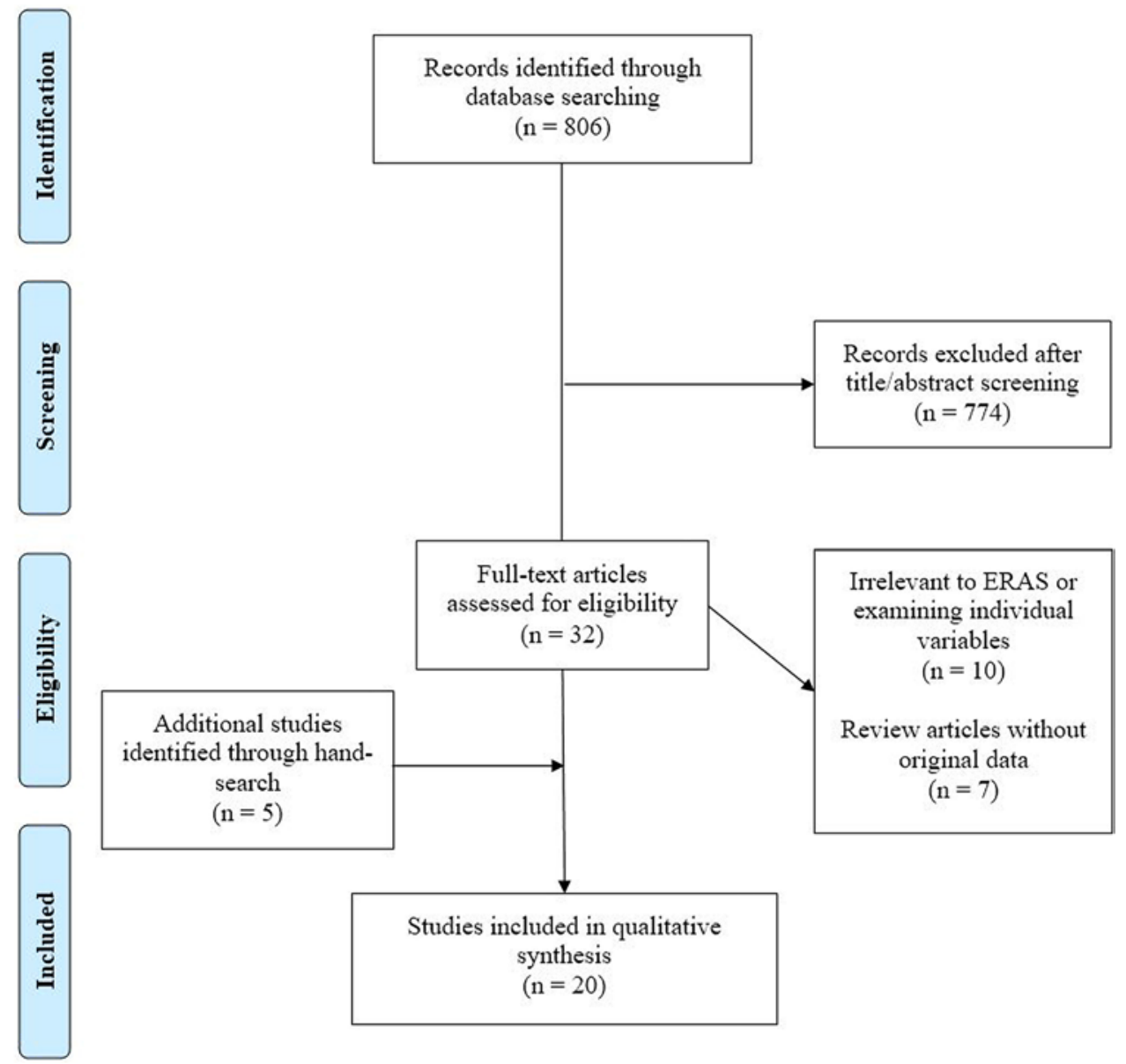

FIG. 2. Flow diagram showing study selection process.

nal data and studies examining only multimodal analgesia or comparing the efficacy of various analgesic medications were excluded. Given that ERAS protocols are designed to address several factors related to perioperative patient care, we did not include studies examining the effect of single variables on outcomes in the absence of an enhanced-recovery framework. A flow diagram detailing methods for selection of studies is presented in Fig. 2. Surveyed outcomes were LOS and rates of readmissions and complications. Data from the included studies were independently extracted by the first author.

Search terms for spine surgery: "spine," "spinal," "lumbar," "cervical," "decompression," "discectomy," "diskectomy," "laminectomy," "laminotomy," "foraminotomy," "facetectomy," "spondylolisthesis," "scoliosis," "kyphoplasty," "vertebroplasty," "laminoplasty," "TLIF," "PLIF," "ALIF," "LLIF," "XLIF," "OLIF," "Smith-Petersen osteotomy," "Smith Petersen osteotomy," "Smith-Peterson osteotomy," "Smith Peterson osteotomy," "Ponte osteotomy," "pedicle subtraction osteotomy," "vertebral column resection," "anterior column realignment." Search terms for ERAS: "fast track," "fast-track," "ERAS," "enhanced recovery protocol," "enhanced recovery pathway," "enhanced recovery program," "enhanced recovery system," "enhanced recovery strategy," "enhanced recovery after surgery," "clinical pathway," "critical pathway," "multi- modal perioperative," "perioperative protocol," "postoperative protocol," "postoperative recovery," "accelerated recovery," "recovery program," "recovery pathway," "multimodal postoperative."

\section{Results}

As enhanced-recovery programs are not currently widely adopted within spine surgery, many investigational studies report their initial approaches to and results of introducing such programs. We categorized these studies as surgery in the lumbar spine, surgeries for correction of scoliosis or deformity, and surgeries of the cervical spine. Combined cervical/lumbar cohorts were included under cervical surgery.

\section{Lumbar Spine}

Soffin et $a .^{46}$ designed an enhanced-recovery pathway for 61 patients undergoing lumbar microdiscectomy or decompression surgery. Preoperative elements of their protocol included patient education, limited fasting, and a carbohydrate beverage 4 hours prior to surgery. Adjunctive nonopioid analgesics and antiemetic prophylaxis were provided. Intravenous fluids and warming were targeted to maintain euvolemia and normothermia. A minimally invasive approach to both surgeries was utilized. Foley 
catheters and drains were avoided. Other components of postoperative management included cessation of intravenous fluids, prompt oral intake, and rapid mobilization. They reported a median LOS of 279 minutes overall. No readmissions within 3 months of surgery were seen.

Wang et al. ${ }^{54}$ proposed an ERAS protocol for 42 patients undergoing lumbar fusion. They did not implement pre- and postoperative elements of the pathway but rather focused on the operative approach and anesthetic considerations. The procedure consisted of a minimally invasive transforaminal lumbar interbody fusion (TLIF) via an endoscopic channel with percutaneous pedicle screw fixation and was performed entirely under conscious sedation and with application of local anesthetic agents. In their series, laryngeal access was not obtained. Foley catheters were not placed, and narcotic medications were not utilized. They reported no intraoperative events related to anesthesia, sedation, or medical events. All patients were ambulatory on either the day of or the day after surgery. A followup study by Wang et al..$^{53}$ retrospectively compared the first 38 patients treated with their ERAS protocol to a group of 15 patients who underwent minimally invasive TLIF prior to the introduction of the enhanced-recovery sequence. Differences between the two groups included the use of general anesthesia and surgery without utilization of the endoscope or the addition of liposomal bupivacaine in the comparison group. Shorter LOS (1.23 vs 3.9 days, $\mathrm{p}=0.009$ ), reduced operative time, less blood loss, fewer complications, and a $15.2 \%$ lower total cost for acute-care hospitalization were observed in the ERAS group.

Although not utilized by them in this series, Wang et al. ${ }^{54}$ provided recommendations regarding other perioperative components of a complete enhanced-recovery pathway, including patient education; nutrition; carbohydrate loading; avoiding routine use of drains and urinary catheterization; targeting euvolemia, normothermia, and normotension; and early postoperative mobilization and enteral nutrition.

Grasu et al. ${ }^{20}$ retrospectively evaluated the efficacy of an enhanced recovery after spine surgery (ERSS) program in 56 patients who underwent spine surgery for metastatic tumors. A variety of open and minimally invasive procedures were utilized. Their pathway included preoperative education and multimodal management of pain and anxiety, carbohydrate loading with limited fasting, euvolemia, normothermia, utilization of minimally invasive techniques when possible, and other elements. Postoperatively, emphasis was placed on early ambulation, rehabilitation, and oral intake. Compared to 41 patients in the preprotocol cohort, those in the ERSS group achieved slightly better average pain control. However, no significant differences were seen with regard to 30-day readmission rates, complications, and LOS (6.3 vs 6.8 days, $\mathrm{p}=0.590$ ). The authors noted that patients with advanced metastatic disease or terminal illness may have prolonged hospitalizations for cancer care unrelated to surgical procedures for which they were admitted.

Nazarenko et al. ${ }^{36}$ applied a fast-track program to 23 patients who underwent microdiscectomy for lumbosacral disc herniation and compared results to 25 patients undergoing the same procedure managed with a standard perioperative algorithm. Central aspects of their fast-track approach were presurgical optimization, patient education emphasizing the patient's role in rehabilitation, early physiotherapy, and goal-directed discharge. Patients in the fasttrack group had a faster recovery and significantly shorter LOS (2.3 vs 3.8 days, $p<0.05$ ). Furthermore, patient satisfaction questionnaires revealed improved pain control and improved overall patient experience in the fast-track group.

Scanlon and Richards ${ }^{43}$ instituted early ambulation and enteral nutrition and optimized anesthetic agents to achieve faster return to baseline in 27 patients undergoing lumbar decompression. Using such a program, they reported same-day discharge without increased 30-day readmissions. Although not including a formal control group in the study design, the investigators reported that patients undergoing this surgery at their institution had historically been admitted for 1-3 days postoperatively.

Fleege et al..$^{14}$ described a fast-track protocol for patients undergoing one- to two-segment stabilization for degenerative lumbar spine pathologies. By ensuring patient education, mobilization on the day of surgery, and aggressive rehabilitation, the investigators were able to reduce hospital LOS from 10.9 to 6.2 days.

Zhang et al ${ }^{56}$ explored the feasibility of enhanced recovery after surgery in patients with lumbar spondylolisthesis undergoing mobile microendoscopic discectomy and TLIF. They found that the ERAS pathway reduced intraoperative bleeding, shortened LOS, improved postoperative pain, and promoted rapid rehabilitation of patients without impacting long-term outcomes.

Bradywood et al. ${ }^{6}$ implemented a care pathway for patients undergoing lumbar fusion. They hypothesized that variation in postoperative management was a significant cause of inefficiency in their healthcare system and created a protocol to address pain control, time points at which to discontinue patient-controlled analgesia (PCA) and Foley catheters, frequency of mobility, and communication between healthcare teams, among other factors. In their analysis of 458 patients, they found that the clinical care pathway decreased mean LOS from 3.9 to 3.4 days $(\mathrm{p}<0.001)$, resulted in a more favorable disposition $(75 \%$ vs $64 \%$ discharged to home, $\mathrm{p}=0.002$ ), and significantly reduced duration of urinary catheterization compared to historical controls. No changes were seen in 30-day readmissions or patient satisfaction data.

\section{Correction of Scoliosis and Spinal Deformity}

A clinical pathway by Muhly et al. ${ }^{35}$ incorporated multimodal analgesia, early mobilization, prompt discontinuation of opioid medications, and other measures for rapid recovery in adolescents undergoing posterior spinal fusion (PSF) for idiopathic scoliosis. Study groups included historical controls $(n=134)$, patients from a transition period when the program was introduced and modified $(n=104)$, and patients in the rapid-recovery pathway $(n=84)$. A decrease in the mean LOS from 5.7 to 4 days and reduced pain scores on postoperative days 0 and 1 were observed after transition into and full implantation of the rapid-recovery pathway. Similar rates of 30-day readmissions were observed.

Gornitzky et al. ${ }^{19}$ compared 58 patients undergoing a 
rapid-recovery pathway after PSF for adolescent idiopathic scoliosis (AIS) to 81 historical controls. Similar to Muhly et al., ${ }^{35}$ they instituted early rehabilitation, transition to oral intake, and prompt discontinuation of drains, catheters, and opioid analgesics. The results showed significantly faster time to urinary catheter removal (1.5 vs 2.0 days) and PCA discontinuation (2.0 vs 3.6 days) and a shorter LOS (3.5 vs 5.0 days) ( $p<0.001$ for all comparisons). Mean daily pain scores were significantly reduced on postoperative days 0 , 1 , and 2 . There was no difference in 30-day readmission rates between the groups.

Chan et al. ${ }^{8}$ examined the feasibility and outcome of an accelerated recovery protocol in 74 adolescents undergoing PSF for correction of idiopathic scoliosis. Fixed discharge criteria were set, and postoperative recovery milestones were established to address the removal of urinary catheters and subfascial drains, as well as timing of dressing changes, mobilization, and PCA discontinuation. Methods to improve postoperative pain management, shorten operative time, and minimize blood loss were implemented. Intraoperative antiemetic prophylaxis was provided. Compared to 33 historical controls, the enhanced-recovery group had a shorter mean LOS (5.2 vs 3.6 days). Only 1 patient failed to meet milestones, and the only complication was a superficial wound infection in 1 patient that was managed on an outpatient basis. No other complications or readmissions were seen within the 4-month follow-up period.

Fletcher et al. ${ }^{15}$ evaluated the efficacy of an accelerated discharge (AD) pathway for patients undergoing PSF for AIS. They compared the AD protocol at one center to a traditional discharge (TD) pathway at a different center. The pathway emphasized early transition to oral pain medications, frequent mobilization with physical therapy, and discharge regardless of a return of bowel function. One hundred five patients were treated by an AD pathway, whereas 45 patients were managed using a TD pathway. The AD group had a $48 \%$ shorter LOS (2.2 vs 4.2 days, $\mathrm{p}<$ 0.0001 ) and significantly fewer total complications than the TD cohort. There was no difference in readmission rates between groups. In their earlier work studying patients with AIS undergoing PSF, Fletcher et al. ${ }^{16}$ compared 279 patients managed with an $\mathrm{AD}$ pathway at one institution to 86 patients treated with a standard discharge pathway at a separate institution. Their results demonstrated that an AD pathway prioritizing early transition to oral pain medications and a solid diet, frequent mobilization with physical therapy, and early removal of drains and urinary catheters resulted in earlier discharge (2.9 vs 4.3 days, $\mathrm{p}<0.0001$ ) and no significant increase in complications.

Sanders et al. ${ }^{42}$ tested an AD pathway for the management of AIS in 90 patients compared to 194 patients managed with a TD pathway. Their pathway incorporated similar measures to those described by Fletcher et al. Shorter mean durations of hospitalization were seen when comparing the two groups (3.7 vs 5.0 days, $\mathrm{p}<0.001$ ). Incidence of complications or readmissions was not increased in the $\mathrm{AD}$ group. Hospital charges for postoperative care were $22 \%$ lower $(\$ 18,360$ vs $\$ 23,640, p<0.0001)$ in the $\mathrm{AD}$ cohort compared to the TD group.

Rao et al. ${ }^{40}$ conducted a retrospective review of adoles- cents who had undergone PSF before and after implementation of pathways to standardize aspects of postoperative care such as pain, management of incisions, nutrition, voiding, and activity. A later revision to the protocol required removal of the Foley and epidural catheters a day earlier. Data were gathered for 51 patients managed before introduction of any protocols, 100 patients who were managed with the first protocol, and 39 patients managed with the second protocol. Clearance for discharge by physical therapy, time to removal of Foley catheter and epidural/PCA, and LOS were significantly decreased in patients managed with the final protocol.

\section{Cervical and Combined Cervical/Lumbar Cohorts}

Sivaganesan et al ${ }^{44}$ performed a retrospective analysis comparing patients who underwent elective lumbar or cervical spine surgery before and after implementation of an ERAS protocol ( $\mathrm{n}=1579$ and 151 for preprotocol and postprotocol, respectively). Procedures included anterior cervical discectomy and fusion (ACDF), microdiscectomy, and laminectomy with or without fusion. Elements of the protocol included appropriate thromboembolism prophylaxis, use of bracing when indicated, bed rest in case of durotomy, multimodal pre- and postoperative pain control, and suitable antibiotic prophylaxis. Early patient mobilization, drain removal, and discharge planning were emphasized. Results demonstrated reduced LOS and 90day complication rates for postprotocol patients. Discharge status, readmission rates, and 3-month patient-reported outcomes were comparable between the two groups. Subgroup analysis demonstrated no significant difference between any outcome measures in patients undergoing cervical surgery, while patients undergoing lumbar surgery in the ERAS pathway had a significantly shorter LOS (2.9 vs 2.5 days, $\mathrm{p}=0.021)$ and fewer complications $(12.8 \%$ vs $3.8 \%, \mathrm{p}=0.002$ ).

Dai et al. ${ }^{11}$ instituted an ERAS protocol for 35 patients undergoing $\mathrm{ACDF}$ and laminoplasty for cervical spondylotic myelopathy. Compared to a control group of 20 patients, the ERAS group had a significantly shorter average LOS (5.62 vs 9.85 days, $\mathrm{p}<0.01)$. Additionally, short-term ( $\leq 30$ day) visual analog scale and Japanese Orthopaedic Association scores were significantly improved in the ERAS cohort.

Venkata and van Dellen ${ }^{50}$ described the implementation of an enhanced-recovery program utilizing extensive patient counseling, preemptive coordination of disposition needs, limitation of opioid medications, emphasis on early mobilization, and avoidance of drains and blood transfusions in patients undergoing open surgery for degenerative lumbar $(\mathrm{n}=187)$ and cervical $(\mathrm{n}=50)$ conditions. The investigators successfully achieved same-day discharge for many patients with few readmissions within 30 days and no long-term complications.

\section{Discussion}

The majority of reviewed studies found that implementation of enhanced-recovery protocols in spine surgery was feasible and associated with a shorter LOS and accelerated return to function without increasing rates of 
complications or readmissions. This benefit was observed across several procedures and patient cohorts. Common elements seen in many enhanced-recovery pathways are the utilization of minimally invasive surgery when possible, use of multimodal analgesia, and early rehabilitation and enteral nutrition.

The use of minimally invasive approaches is increasingly common in spine surgery. ${ }^{24}$ Several of the reviewed studies incorporated these techniques as part of their enhanced-recovery pathways. Numerous systematic reviews and meta-analyses ${ }^{18,34,39}$ have found minimally invasive spine surgeries to be associated with shorter LOS, while having comparable long-term outcomes. Nevertheless, surgical planning must consider other variables besides recovery time and ultimately is to be decided on a caseby-case basis.

Although several of the aforementioned ERAS protocols differed in the exact analgesic regimen, multimodal pain control was a common theme. In their article on perioperative principles related to complex spine surgery, Lamperti et al. ${ }^{27}$ listed and reviewed evidence surrounding several nonopioid medications that may be utilized to decrease postoperative pain. Given the extensive side effect profile of opioid medications, the use of adjunct analgesics whenever possible is encouraged.

There is evidence that early oral intake after surgery is safe and may hasten return of bowel function and reduce the duration of hospitalization in other surgical disciplines. $.17,23,37,55$ While evidence targeted specifically at spine surgery is lacking, acceleration of postoperative enteral nutrition was a cornerstone of many of the aforementioned enhanced-recovery programs and is routinely incorporated in ERAS pathways in other surgical fields. ${ }^{33}$

The benefits of early mobilization following spine surgery and other procedures were reviewed by Epstein. ${ }^{12}$ Many studies revealed reduced rates of infections and medical complications along with decreased average LOS after instituting early-mobilization protocols. In addition to hastening a return to baseline level of function, accelerated ambulation and rehabilitation also serve to emphasize the patient's role in recovery.

The majority of studies we found examined the benefits of ERAS for degenerative disease and stenosis of the lumbar spine. While these are certainly among the most commonly encountered pathologies, we especially note a lack of investigations addressing adults undergoing reconstruction for spinal deformity and degenerative scoliosis. This is an important area of further research given the rising frequency of and tremendous costs associated with correction of adult spinal deformity. ${ }^{58}$ Lastly, no studies addressed intradural lesions of the spinal cord itself; these surgeries may also be amenable to the application of enhanced-recovery principles.

Despite the increasing rates of spine procedures, ${ }^{26}$ standardized criteria for perioperative management for specific surgeries are lacking. This variation in practice may be a cause of extended hospitalizations in patients undergoing lumbar and cervical spine surgeries. ${ }^{1,2}$ Given their nature as evidence-based platforms intended to streamline care and reduce waste, enhanced-recovery strategies enforce the use of standardized principles to minimize variations in practice among surgical teams. Enforcing consistency of postoperative care may underlie some of the benefit imparted by the adoption of ERAS programs.

There are limitations to the reviewed data and our study that should be noted. As there is no technical definition for an ERAS protocol, there is a risk of bias at the reporting level when deciding which studies to include. A quantitative synthesis was not performed due to heterogeneity in the reviewed studies with regard to design, populations, procedures, methods, and outcomes. Not all studies had formal control groups. Some outcomes, such as pain control and cost, were not reported across all studies. We therefore focused on outcomes that were most likely to be consistently reported. Lastly, many studies compared rates of complications between intervention and control groups; however, the exact definition of this outcome varied among studies.

\section{Conclusions}

Current evidence surrounding the use of ERAS protocols in spine surgery is largely restricted to retrospective reviews of nonrandomized data sets and preliminary cohort studies lacking formal control groups. These introductory studies have demonstrated the potential of enhanced-recovery protocols to reduce LOS, accelerate return of function, minimize postoperative pain, and save costs. Rigorous RCTs may serve to provide robust evidence and establish the efficacy of enhanced-recovery programs for particular patient populations and procedures within spine surgery. Indeed, Ali et al. ${ }^{3}$ provided a detailed protocol for a future multicenter RCT to test improvements in healthcare quality associated with implementation of an ERAS pathway in patients undergoing elective spine surgeries. Given the nature of ERAS programs as quality improvement initiatives, continuous data-driven reassessment and optimization are integral to ensure excellent outcomes.

\section{References}

1. Adogwa O, Desai SA, Vuong VD, Lilly DT, Ouyang B, Davison M, et al: Extended length of stay in elderly patients after lumbar decompression and fusion surgery may not be attributable to baseline illness severity or postoperative complications. World Neurosurg 116:e996-e1001, 2018

2. Adogwa O, Lilly DT, Vuong VD, Desai SA, Ouyang B, Khalid S, et al: Extended length of stay in elderly patients after anterior cervical discectomy and fusion is not attributable to baseline illness severity or postoperative complications. World Neurosurg 115:e552-e557, 2018

3. Ali ZS, Ma TS, Ozturk AK, Malhotra NR, Schuster JM, Marcotte PJ, et al: Pre-optimization of spinal surgery patients: development of a neurosurgical enhanced recovery after surgery (ERAS) protocol. Clin Neurol Neurosurg 164:142-153, 2018

4. Bannister M, Ah-See KW: Enhanced recovery programmes in head and neck surgery: systematic review. J Laryngol Otol 129:416-420, 2015

5. Barton JG: Enhanced recovery pathways in pancreatic surgery. Surg Clin North Am 96:1301-1312, 2016

6. Bradywood A, Farrokhi F, Williams B, Kowalczyk M, Blackmore CC: Reduction of inpatient hospital length of stay in lumbar fusion patients with implementation of an evidence- 
based clinical care pathway. Spine (Phila Pa 1976) 42:169_ 176, 2017

7. Brown JK, Singh K, Dumitru R, Chan E, Kim MP: The benefits of Enhanced Recovery After Surgery programs and their application in cardiothoracic surgery. Methodist Debakey Cardiovasc J 14:77-88, 2018

8. Chan CYW, Loo SF, Ong JY, Lisitha KA, Hasan MS, Lee $\mathrm{CK}$, et al: Feasibility and outcome of an accelerated recovery protocol in Asian adolescent idiopathic scoliosis patients. Spine (Phila Pa 1976) 42:E1415-E1422, 2017

9. Charoenkwan K, Matovinovic E: Early versus delayed oral fluids and food for reducing complications after major abdominal gynaecologic surgery. Cochrane Database Syst $\operatorname{Rev}(12): C D 004508,2014$

10. Currie A, Burch J, Jenkins JT, Faiz O, Kennedy RH, Ljungqvist $\mathrm{O}$, et al: The impact of enhanced recovery protocol compliance on elective colorectal cancer resection: results from an international registry. Ann Surg 261:1153-1159, 2015

11. Dai B, Gao P, Dong QR, Wang YM, Chen D, Shen YC, et al: [Clinical study of the application of enhanced recovery after surgery in cervical spondylotic myelopathy.] Zhongguo Gu Shang 31:740-745, 2018 (Chinese)

12. Epstein NE: A review article on the benefits of early mobilization following spinal surgery and other medical/surgical procedures. Surg Neurol Int 5 (Suppl 3):S66-S73, 2014

13. Fearon $\mathrm{KCH}$, Ljungqvist $\mathrm{O}$, Von Meyenfeldt M, Revhaug A, Dejong CHC, Lassen K, et al: Enhanced recovery after surgery: a consensus review of clinical care for patients undergoing colonic resection. Clin Nutr 24:466-477, 2005

14. Fleege C, Arabmotlagh M, Almajali A, Rauschmann M: [Pre- and postoperative fast-track treatment concepts in spinal surgery: patient information and patient cooperation.] Orthopade 43:1062-1064, 1066-1069, 2014 (Ger)

15. Fletcher ND, Andras LM, Lazarus DE, Owen RJ, Geddes BJ, Cao J, et al: Use of a novel pathway for early discharge was associated with a $48 \%$ shorter length of stay after posterior spinal fusion for adolescent idiopathic scoliosis. J Pediatr Orthop 37:92-97, 2017

16. Fletcher ND, Shourbaji N, Mitchell PM, Oswald TS, Devito DP, Bruce RW: Clinical and economic implications of early discharge following posterior spinal fusion for adolescent idiopathic scoliosis. J Child Orthop 8:257-263, 2014

17. Fujii T, Morita H, Sutoh T, Yajima R, Yamaguchi S, Tsutsumi $\mathrm{S}$, et al: Benefit of oral feeding as early as one day after elective surgery for colorectal cancer: oral feeding on first versus second postoperative day. Int Surg 99:211-215, 2014

18. Goldstein CL, Macwan K, Sundararajan K, Rampersaud YR: Perioperative outcomes and adverse events of minimally invasive versus open posterior lumbar fusion: meta-analysis and systematic review. J Neurosurg Spine 24:416-427, 2016

19. Gornitzky AL, Flynn JM, Muhly WT, Sankar WN: A rapid recovery pathway for adolescent idiopathic scoliosis that improves pain control and reduces time to inpatient recovery after posterior spinal fusion. Spine Deform 4:288-295, 2016

20. Grasu RM, Cata JP, Dang AQ, Tatsui CE, Rhines LD, Hagan $\mathrm{KB}$, et al: Implementation of an Enhanced Recovery After Spine Surgery program at a large cancer center: a preliminary analysis. J Neurosurg Spine 29:588-598, 2018

21. Greco M, Capretti G, Beretta L, Gemma M, Pecorelli N, Braga M: Enhanced recovery program in colorectal surgery: a meta-analysis of randomized controlled trials. World J Surg 38:1531-1541, 2014

22. Hagan KB, Bhavsar S, Raza SM, Arnold B, Arunkumar R, Dang A, et al: Enhanced recovery after surgery for oncological craniotomies. J Clin Neurosci 24:10-16, 2016

23. Hoshi T, Yamashita S, Tanaka M, Motokawa K, Toyooka H: Early oral intake after arthroscopic surgery under spinal anesthesia. J Anesth 13:205-208, 1999
24. Huang TJ, Kim KT, Nakamura H, Yeung AT, Zeng J: The state of the art in minimally invasive spine surgery. Biomed Res Int 2017:6194016, 2017

25. Kleppe KL, Greenberg JA: Enhanced recovery after surgery protocols: rationale and components. Surg Clin North Am 98:499-509, 2018

26. Kobayashi K, Ando K, Nishida Y, Ishiguro N, Imagama S: Epidemiological trends in spine surgery over 10 years in a multicenter database. Eur Spine J 27:1698-1703, 2018

27. Lamperti M, Tufegdzic B, Avitsian R: Management of complex spine surgery. Curr Opin Anaesthesiol 30:551-556, 2017

28. Lee L, Feldman LS: Enhanced recovery after surgery: economic impact and value. Surg Clin North Am 98:11371148,2018

29. Lee L, Mata J, Ghitulescu GA, Boutros M, Charlebois P, Stein B, et al: Cost-effectiveness of enhanced recovery versus conventional perioperative management for colorectal surgery. Ann Surg 262:1026-1033, 2015

30. Lillemoe HA, Aloia TA: Enhanced recovery after surgery: hepatobiliary. Surg Clin North Am 98:1251-1264, 2018

31. Liu JY, Wick EC: Enhanced recovery after surgery and effects on quality metrics. Surg Clin North Am 98:1119-1127, 2018

32. Ljungqvist O: Jonathan E. Rhoads lecture 2011: insulin resistance and enhanced recovery after surgery. JPEN J Parenter Enteral Nutr 36:389-398, 2012

33. Ljungqvist O, Scott M, Fearon KC: Enhanced Recovery After Surgery: a review. JAMA Surg 152:292-298, 2017

34. Lu VM, Kerezoudis P, Gilder HE, McCutcheon BA, Phan K, Bydon M: Minimally invasive surgery versus open surgery spinal fusion for spondylolisthesis: a systematic review and meta-analysis. Spine (Phila Pa 1976) 42:E177-E185, 2017

35. Muhly WT, Sankar WN, Ryan K, Norton A, Maxwell LG, DiMaggio T, et al: Rapid recovery pathway after spinal fusion for idiopathic scoliosis. Pediatrics 137:e20151568, 2016

36. Nazarenko AG, Konovalov NA, Krut'ko AV, Zamiro TN, Geroeva IB, Gubaydullin RR, et al: [Postoperative applications of the fast track technology in patients with herniated intervertebral discs of the lumbosacral spine.] Zh Vopr Neirokhir Im N N Burdenko 80:5-12, 2016 (Russian)

37. Nematihonar B, Salimi S, Noorian V, Samsami M: Early versus delayed (traditional) postoperative oral feeding in patients undergoing colorectal anastomosis. Adv Biomed Res 7:30, 2018

38. Pędziwiatr M, Mavrikis J, Witowski J, Adamos A, Major P, Nowakowski M, et al: Current status of Enhanced Recovery After Surgery (ERAS) protocol in gastrointestinal surgery. Med Oncol 35:95, 2018

39. Phan K, Mobbs RJ: Minimally invasive versus open laminectomy for lumbar stenosis: a systematic review and metaanalysis. Spine (Phila Pa 1976) 41:E91-E100, 2016

40. Rao RR, Hayes M, Lewis C, Hensinger RN, Farley FA, Li Y, et al: Mapping the road to recovery: shorter stays and satisfied patients in posterior spinal fusion. J Pediatr Orthop 37:e536-e542, 2017

41. Saidian A, Nix JW: Enhanced recovery after surgery: urology. Surg Clin North Am 98:1265-1274, 2018

42. Sanders AE, Andras LM, Sousa T, Kissinger C, Cucchiaro G, Skaggs DL: Accelerated discharge protocol for posterior spinal fusion patients with adolescent idiopathic scoliosis decreases hospital postoperative charges 22\%. Spine (Phila Pa 1976) 42:92-97, 2017

43. Scanlon J, Richards B: Development of a same day laminectomy program. J Perianesth Nurs 19:84-88, 2004

44. Sivaganesan A, Wick JB, Chotai S, Cherkesky C, Stephens BF, Devin CJ: Perioperative protocol for elective spine surgery is associated with reduced length of stay and complications. J Am Acad Orthop Surg [epub ahead of print], 2018 
45. Smith HJ, Leath CA III, Straughn JM Jr: Enhanced recovery after surgery in surgical specialties: gynecologic oncology. Surg Clin North Am 98:1275-1285, 2018

46. Soffin EM, Vaishnav AS, Wetmore D, Barber L, Hill P, Gang $\mathrm{CH}$, et al: Design and implementation of an enhanced recovery after surgery (ERAS) program for minimally invasive lumbar decompression spine surgery: initial experience. Spine (Phila Pa 1976) [epub ahead of print], 2018

47. Thacker J: Overview of enhanced recovery after surgery: the evolution and adoption of enhanced recovery after surgery in North America. Surg Clin North Am 98:1109-1117, 2018

48. Thiele RH, Rea KM, Turrentine FE, Friel CM, Hassinger TE, McMurry TL, et al: Standardization of care: impact of an enhanced recovery protocol on length of stay, complications, and direct costs after colorectal surgery. J Am Coll Surg 220:430-443, 2015

49. Tiernan JP, Liska D: Enhanced recovery after surgery: recent developments in colorectal surgery. Surg Clin North Am 98:1241-1249, 2018

50. Venkata HK, van Dellen JR: A perspective on the use of an enhanced recovery program in open, non-instrumented day surgery for degenerative lumbar and cervical spinal conditions. J Neurosurg Sci 62:245-254, 2018

51. Visioni A, Shah R, Gabriel E, Attwood K, Kukar M, Nurkin $\mathrm{S}$ : Enhanced recovery after surgery for noncolorectal surgery?: a systematic review and meta-analysis of major abdominal surgery. Ann Surg 267:57-65, 2018

52. Wainwright TW, Immins T, Middleton RG: Enhanced Recovery after Surgery (ERAS) and its applicability for major spine surgery. Best Pract Res Clin Anaesthesiol 30:91-102, 2016

53. Wang MY, Chang HK, Grossman J: Reduced acute care costs with the ERAS ${ }^{\circledR}$ minimally invasive transforaminal lumbar interbody fusion compared with conventional minimally invasive transforaminal lumbar interbody fusion. Neurosurgery 83:827-834, 2018

54. Wang MY, Chang PY, Grossman J: Development of an Enhanced Recovery After Surgery (ERAS) approach for lumbar spinal fusion. J Neurosurg Spine 26:411-418, 2017
55. Yin X, Ye L, Zhao L, Li L, Song J: Early versus delayed postoperative oral hydration after general anesthesia: a prospective randomized trial. Int J Clin Exp Med 7:3491-3496, 2014

56. Zhang CH, Yan BS, Xu BS, Ma XL, Yang Q, Liu Y, et al: [Study on feasibility of enhanced recovery after surgery combined with mobile microendoscopic discectomy-transforaminal lumbar interbody fusion in the treatment of lumbar spondylolisthesis.] Zhonghua Yi Xue Za Zhi 97:1790-1795, 2017 (Chinese)

57. Zhu S, Qian W, Jiang C, Ye C, Chen X: Enhanced recovery after surgery for hip and knee arthroplasty: a systematic review and meta-analysis. Postgrad Med J 93:736-742, 2017

58. Zygourakis CC, Liu CY, Keefe M, Moriates C, Ratliff J, Dudley RA, et al: Analysis of national rates, cost, and sources of cost variation in adult spinal deformity. Neurosurgery 82:378-387, 2018

\section{Disclosures}

The authors report no conflict of interest concerning the materials or methods used in this study or the findings specified in this paper.

\section{Author Contributions}

Conception and design: Elsarrag, Soldozy. Acquisition of data: Elsarrag. Analysis and interpretation of data: Elsarrag, Patel. Drafting the article: Elsarrag, Soldozy, Patel. Critically revising the article: Kalani, Elsarrag, Soldozy, Patel. Reviewed submitted version of manuscript: all authors. Approved the final version of the manuscript on behalf of all authors: Kalani. Administrative/ technical/material support: Kalani, Norat, Sokolowski, Park, Tvrdik. Study supervision: Kalani, Norat, Sokolowski, Park, Tvrdik.

\section{Correspondence}

M. Yashar S. Kalani: University of Virginia Health System, Charlottesville, VA.kalani@virginia.edu. 